\title{
DISCRETE NON-LOCAL ABSORBING BOUNDARY CONDITION FOR EXTERIOR PROBLEMS GOVERNED BY HELMHOLTZ EQUATION
}

\author{
RUPERTO P. BONET*,1, NORBERTO NIGRO, MARIO A. STORTI AND \\ SERGIO R. IDELSOHN \\ Mechanic Technology Group, INTEC, Güemes 3450, 3000 Santa Fe, Argentina
}

\begin{abstract}
SUMMARY
The finite element method is employed to approximate the solutions of the Helmholtz equation for water wave radiation and scattering in an unbounded domain. A discrete, non-local and non-reflecting boundary condition is specified at an artificial external boundary by the DNL method, yielding an equivalent problem that is solved in a bounded domain. This procedure formulates a boundary value problem in a bounded region by imposing a relation in the discrete medium between the nodal values at the two last layers. For plane geometry, this relation can be found by straightforward eigenvalue decomposition. For circular geometry, the plane condition is applied at the external layer and this condition is condensed through a structured annular region, resulting in a condition at an inner radius. Exterior problems with a bounded internal physical obstacle are considered. It is well-known that these kind of problems are well-posed, and have a unique solution. Numerical studies based on standard Galerkin methodology examine the dependence of the DNL condition with respect to the circular annular region width. The DNL condition is compared with local boundary conditions of several orders. Numerical examples confirm the important improvement in accuracy obtained by the DNL method over standard conditions. Copyright (C) 1999 John Wiley \& Sons, Ltd.
\end{abstract}

KEY WORDS: absorbing boundary condition; Helmholtz; discrete; non-local radiation; scattering

\section{INTRODUCTION}

Water waves elliptic propagation models governed by the Helmholtz equation with variable refraction-diffraction index $k=k(x, y)$ [1,2], have received a wide acceptance for performing engineering simulations of water waves propagation over arbitrary bathymetry and in complex coastal domains.

Numerical solutions of the Helmholtz equation in exterior domains have been sought primarily via techniques that are based on the Helmholtz integral representations of the problem, relating quantities on the physical boundary of the problem [1]. Such formulations are based in the consideration that the bathymetry must be constant in the exterior domain. These formulations are obtained by using fundamental solutions as weighting functions and employing Green's theorem, a procedure that typically is restricted to linear, isotropic and

* Correspondence to: Mechanic Technology Group, INTEC, Güemes 3450, 3000 Santa Fe, Argentina. E-mail: rbonet@venus.unl.edu.ar

${ }^{1}$ Tel.: + 5442 559175; fax: + 5442550944 .

CCC 0271-2091/99/050605-17\$17.50

Received September 1997

Copyright (C) 1999 John Wiley \& Sons, Ltd.

Revised February 1998 
homogeneous problems, with the benefit of a priori satisfaction of the radiation condition at infinity, and the advantage of seeking solutions over a domain that is one dimension lower than the original form of the problem [1,3]. It is well-known that this kind of method has difficulties in its implementation, since the system matrix can be ill-conditioned.

An alternative to these formulations is the selection of exponential shape functions in the so-called infinite elements technique [4]. Other approaches convert the boundary value problem to formulations that are defined over bounded regions, by introducing an artificial external boundary with appropriate boundary conditions. Proper representation of the radiation condition then becomes the crucial issue. One approximation to the radiation condition was obtained by employing an asymptotic expansion of the far-field solution to generate a sequence of local boundary operators [5-8]. In this direction, various parabolic approximations to the Helmholtz equation (with variable coefficients) [1,2] have been proposed as boundary conditions for the scattered waves by Radder (1979), Dalrymple and Martin [6], Booij [9], Kirby [10], BingYi Xu et al. [11]. These parabolic equations can accommodate waves exiting through a larger, but still limited, aperture. In addition, the intrinsic of the parabolic equation requires, a priori, the selection of the 'dominant' direction for the exiting waves (which may not be known) and the placement of the artificial boundary in a perpendicular direction to it. This can be cumbersome in domains of complex shape, particularly if several incident wave directions are to be treated.

Once a boundary value problem is formulated in a bounded region, finite element methods may be employed for computation, taking advantage of the wide range of applicability and rich mathematical structure inherent in these techniques.

In the following, formulations for a bounded computation domain, which are derived by the

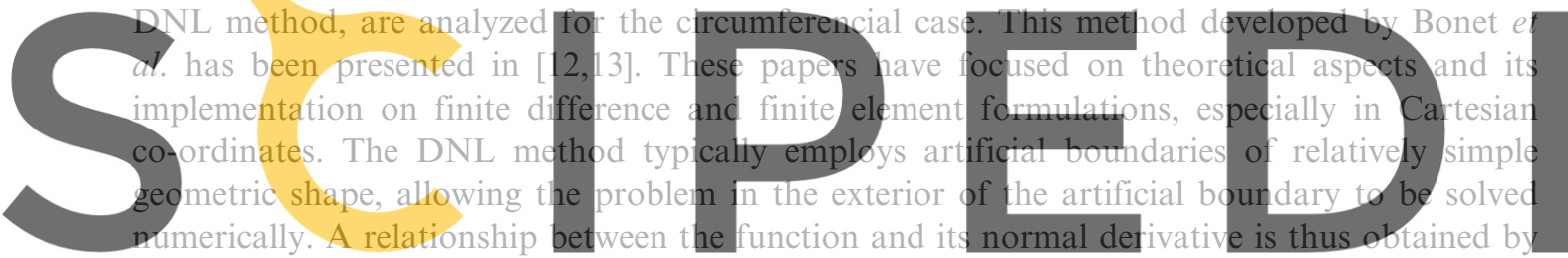

means of the relationship between the nodal function value belonging to the artificial

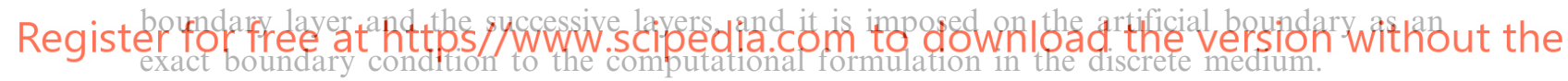

In the DNL formulation, all the operations are developed in the discrete medium, in

contrast to the DtN formulation (Givoli [7], Keller [14,15], Harari [8,16]) and the pseudo-spectral approach obtained by Chen and Philip [17], who solved the exterior problem in analytical form using a series expansion.

In this paper, the DNL formulation is developed in circumferential co-ordinates as an extension from the DNL formulation in rectangular Cartesian co-ordinates [13], by means of a procedure called 'condensation'. In this process, the DNL boundary condition in the far-field (where curvature effects can be neglected) is first obtained, and then, the discretized Helmholtz equation for each radial layer is solved successively, including curvature effects. By means of this procedure, the exterior problem associated with the Helmholtz equation is solved numerically, and a DNL boundary condition over the artificial boundary is obtained.

Several numerical tests will be presented in order to show the performance of the proposed method and the improvement with using the DNL boundary condition with respect to the local radiation boundary conditions of several orders. 


\section{PROBLEM FORMULATION}

The scattering problem produced when an incident wave is reflected from a scatterer is considered. Assuming the incident wave field is time-harmonic, with temporal dependence $\mathrm{e}^{-i \omega t}$, the goal is to solve

$$
\begin{aligned}
& \Delta \phi^{\mathrm{s}}+k^{2} \phi^{\mathrm{s}}=0 \quad \text { in } \Omega^{\infty}, \\
& \phi^{\mathrm{s}}=\bar{\phi}^{\mathrm{s}} \quad \text { on } \Gamma_{g}, \\
& \frac{\partial \phi^{\mathrm{s}}}{\partial \boldsymbol{n}}=-\frac{\partial \phi^{\mathrm{i}}}{\partial \boldsymbol{n}} \text { on } \Gamma_{h}, \\
& \lim _{r \rightarrow \infty} \sqrt{k r}\left(\frac{\partial}{\partial r}-i k\right) \phi^{\mathrm{s}}=0 \quad \text { uniformly in } \theta,
\end{aligned}
$$

where $C$ is the phase velocity, $k=\omega / C \geq 0$ is the wavenumber. $\Gamma$ is the boundary of the scatterer, such that $\Gamma$ admits the partition $\Gamma=\overline{\Gamma_{g} \cup \Gamma_{h}}$, where $\Gamma_{g} \cap \Gamma_{h}=\varnothing$, $\Omega^{\infty}$ is the (unbounded) exterior of the scatterer and $\phi^{i}$ is the incoming wave, $n$ is the outward normal on $\Gamma$ and $r$ is the distance from the origin.

The fourth equation in (1) is the Sommerfeld radiation condition. The radiation condition discards solutions with ingoing waves at infinity and requires that the outgoing energy flux at infinity must be positive.

The basic idea for the reformulation of this problem on a bounded domain is to introduce an artificial boundary $\mathscr{B}$, and solve a new problem on $\Omega$, the computational domain (Figure 1). The new boundary problem obtained is
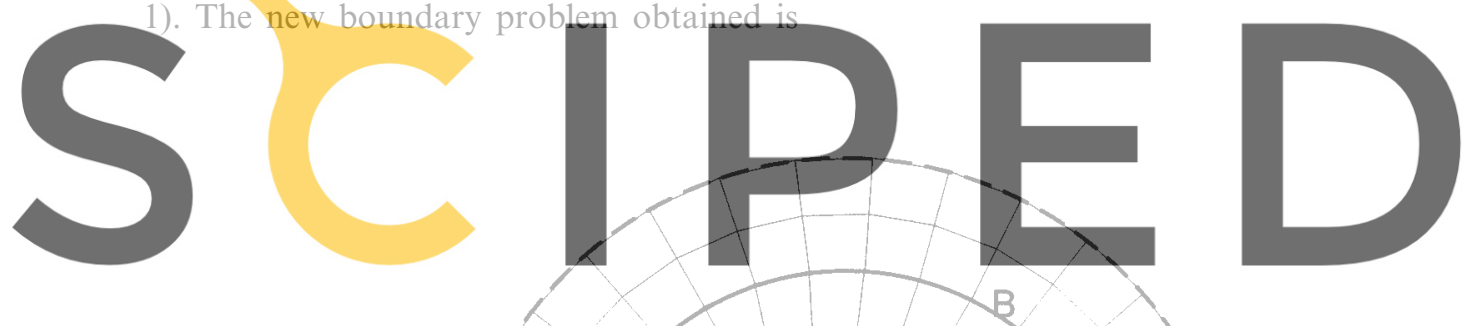

Register for free at https//www.scipedia.com to download the version without the

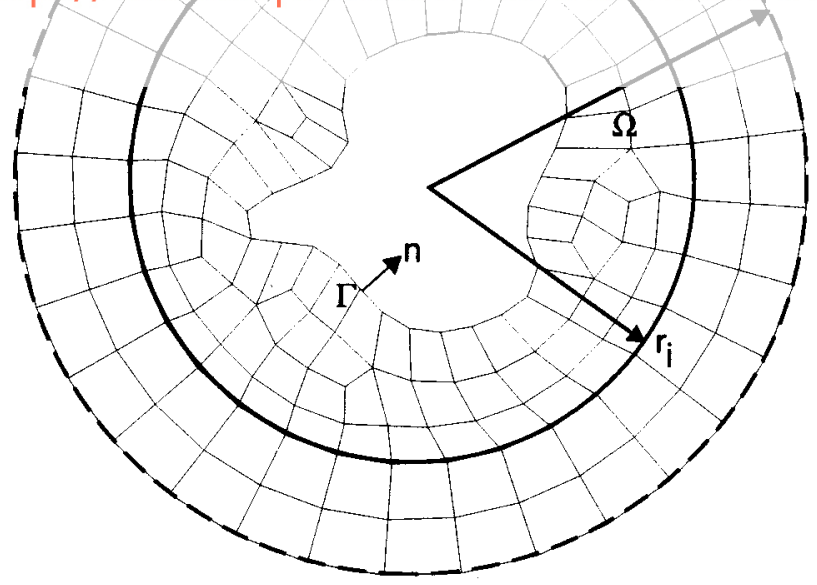

Figure 1. A model domain for radiation and scattering problem. 

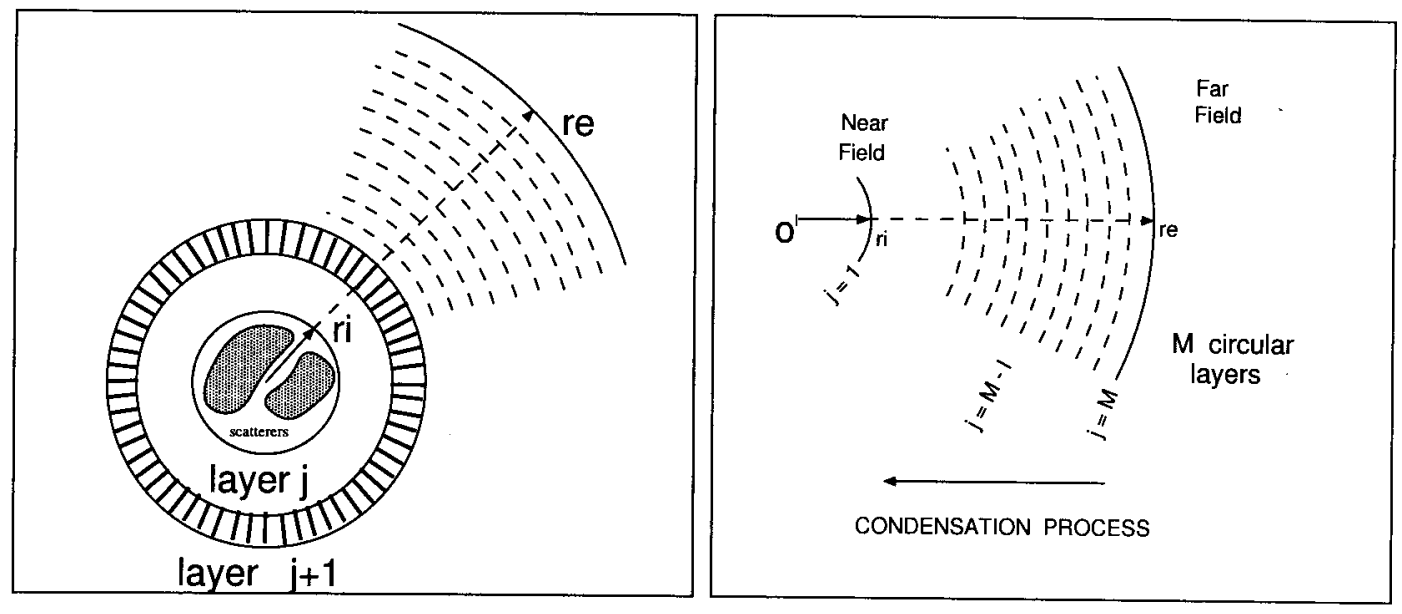

Figure 2. Circular DNL method; sketch on the scattering process by the DNL method (left); condensation process (right).
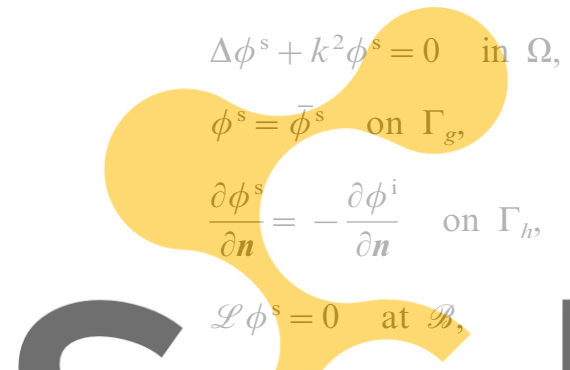

where $\mathscr{L}$ is an as yet undeterm

boundary, a circumference

gomputational domain. This curve encloses the comput

The artificial boundary con

boundary condition; however, this problem is not completely solved yet.

first- or second-order absorbing boundary condition at $\mathscr{B}$ produces a certain amount of

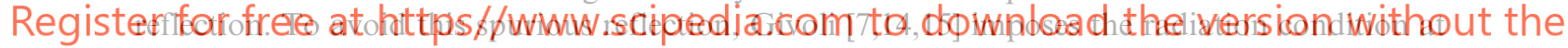

infinity by means of the DtN method, which has been developed by Harari [8,16] for acoustic

problems. This boundary condition is exact and non-reflecting, but the bases system must be selected according to the space dimension of the problem at hands. It is easily implemented in a finite element method [16].

In contrast to the methods described early, a discrete formulation to impose the radiation boundary condition at infinity in a numerical scheme can be developed directly.

A discrete non-reflecting boundary condition $[12,13]$ has been devised in finite differences. The boundary condition is non-local on $\mathscr{B}$. Now, it will be shown how to combine this boundary condition with the finite element method, in order to solve a bounded problem on a truncated domain $\Omega$. Details about the derivation of this procedure are developed in the following sections.

\section{THE DNL FORMULATION}

The circumferential DNL procedure is based on the full solution of the exterior problem governed by the discretized Helmholtz operator with constant refraction index. For this, the 
unbounded domain (in the external region to $\Omega$ (see Figure 1)) is subdivided into two parts, a bounded region (the annular region $r_{\mathrm{i}} \leq r<r_{\mathrm{e}},-\pi \leq \theta \leq \pi$ (see Figure 2)) and an unbounded semi-infinite region (the region $r \geq r_{\mathrm{e}}$ ). Figure 2 (left) shows the scatterers surrounded by a circle of radius $r_{\mathrm{i}}$, located at the artificial boundary (in the near-field) and the successive circles ( $\bigcirc$ layers) until the circle $r=r_{\mathrm{e}}$ (in the far-field), which is made sufficiently large such that the influence of curvature can be neglected. In contrast to Thatcher's method $[18,19]$, the DNL method uses a discretization with quadrangular linear elements (see layer $j$ in Figure 2), resulting an extremely easy implementation. The crucial issue is first the adequate representation of the scattered wave field in the far-field $\left(r \geq r_{\mathrm{e}}\right)$, and after, in the near-field (where the artificial boundary of the computational domain is located $\left.\left(r=r_{\mathrm{i}}\right)\right)$.

Now, a procedure performing the partial discretization of the Helmholtz equation (1) in polar co-ordinates, with quadrangular linear elements will be briefly described. Integrating in the transversal direction $\theta$, a second-order differential equation system in $r$ is obtained of the form:

$$
\mathbf{I} \ddot{\phi}+\frac{1}{r} \mathbf{I} \dot{\phi}-\frac{1}{r^{2}} \mathbf{M}^{-1} \mathbf{K} \phi+k^{2} \mathbf{I} \phi=\overline{0},
$$

where $\phi=\phi^{\mathrm{s}}, \phi=\mathrm{d} \phi^{\mathrm{s}} / \mathrm{d} r, \mathbf{M}$ and $\mathbb{K}$ are the mass and stiffness assembled matrices respectively, and $\mathbf{I}$ is the identity matrix. Discretization of Equation (3) in $r$ can be done by the finite difference method or the finite element method. Independently of the partial discretization procedure employed, the corresponding discrete equation for the layer $j$ has the form:

$$
\mathbf{C}^{j} \phi^{j-1}+\mathbf{B}^{j} \phi^{j}+\mathbf{A}^{j} \phi^{j+1}=0,
$$

where $\phi^{j}$ is the vector containing potential values for the nodes on layer $j$. Note that, unlike the rectangular geometry case, here $\mathrm{A}^{j}, \mathbf{B}^{\mathrm{B}}$ and $\mathrm{C}^{j}$ are different matrices at successive layers, duc
3. $r^{j}$ factors. DNL method in the far-field
To obtain the DNL condition in the near-field, it is necessary to obtain the DNL condition
in the far-field, where the curvature effects can be neglected. This layer is denoted by $j=M$ (see Figure 2(b)). For the layer $j=M$, Equation (4) reduces to

The $\mathbf{A}^{M}$ and $\mathbb{B}^{M}$ matrices are real and cyclic in virtue of the periodicity. Their dimensions are $N_{\text {lay }} \times N_{\text {lag. }}$. Consider that the $\mathrm{A}^{M}$ and $\mathbb{B}^{M}$ matrices remain almost constant for the layer $j \geq M$ (in the semi-infinite region $r \geq r_{\mathrm{e}}$ ), and it allows the application of the planar DNL method [13]. Then, $\mathbf{A}^{M}=\mathbf{A}$ and $\mathbf{B}^{M}=\mathbf{B}$ for the layer $j \geq M$.

For the real matrix $\mathbf{A}^{-1} \mathbf{B}$, there is an orthogonal transformation $V$, such that

$$
\mathbf{A}^{-1} \mathbf{B}=V \mathbf{\Lambda} V^{-1},
$$

where $\boldsymbol{\Lambda}=\operatorname{diag}\left(\lambda_{1}, \lambda_{2}, \ldots, \lambda_{N_{\text {lay }}}\right)$ is a diagonal matrix formed by the eigenvalues of the $\mathbf{A}^{-1} \mathbf{B}$ matrix and $V$ is the eigenvector system of $\mathbf{A}^{-1} \mathbf{B}$.

By means of the non-singular transformation

$$
\phi_{l}^{j}=\sum_{i=1}^{N_{\mathrm{lay}}} V_{l, i}\left(c_{i}^{+} \mu_{i}^{+j}+c_{i}^{-} \mu_{i}^{-j}\right) \quad \text { with } l=1,2, \ldots, N_{\mathrm{lay}},
$$

the scattered wave field is split in 'forward' and 'backward' propagation modes, where $\mu_{i}^{ \pm}$is the characteristic equation solution: 


$$
\left(\mu_{i}^{2}+\lambda_{i} \mu_{i}+1\right)=0 .
$$

In previous work, the propagation modes were characterized, as for each $\lambda_{i}$, if the roots $\mu_{1}$ and $\mu_{2}$ of Equation (8) satisfy $\left|\mu_{1}\right|\left|\mu_{2}\right|=1$, then two possibilities arise. If $\left|\mu_{1}\right|<1<\left|\mu_{2}\right|$, you can define $\mu_{i}^{+}=\mu_{1}$ and $\mu_{i}^{-}=\mu_{2}$, whereas if $\left|\mu_{1}\right|=\left|\mu_{2}\right|=1$, the selection is done on the basis of the group velocity, which results in $\operatorname{Im}\left(\mu_{i}^{+}\right)>0[12,13]$.

The matrix $\mathbf{G}$ is denoted as $\mathbf{G}=\operatorname{diag}\left(\mu_{1}(\lambda), \ldots, \mu_{N_{\text {lay }}}(\lambda)\right)$ and based on Equation (7), is easy to prove that Equation (5) is satisfied exactly for 'forward' propagation modes by means of the relation

$$
\left(\phi^{+}\right)^{j+1}=\mathbf{F}\left(\phi^{+}\right)^{j},
$$

such that the matrix $\mathbf{F}$, called the planar DNL matrix, can be given by $\mathbf{F}=V \mathbf{G} V^{-1}$. Then, by means of this procedure developed for the rectangular Cartesian co-ordinates [12,13], you can obtain the corresponding DNL matrix $\mathbf{F}^{M}=\mathbf{F}$ for the layer $j=M$,

$$
\left(\phi^{+}\right)^{M+1}=\mathbf{F}^{M}\left(\phi^{+}\right)^{M} .
$$

\subsection{Derivation of the DNL method in the near-field}

The derivation of the DNL method in the near-field is obtained by means of the recursive process of calculus from the far-field $(j=M)$ to the near field $(j=1)$ (see Figure 2 (right)).

Substituting Equation (10) into Equation (4) for the layer $j=M$, and solving (4) for $\left(\phi^{+}\right)^{M}$, you obtain the DNL matrix for the layer $j=M-1$ :

$$
\mathbf{F}^{M-1}=-\left(\mathbf{A}^{M} \mathbf{F}^{M}+\mathbf{B}^{M}\right)^{-1} \mathbf{C}^{M} .
$$

Repeating this calculation recursively from layer $j=M-1$ to $j=1$ (the layer corresponding to

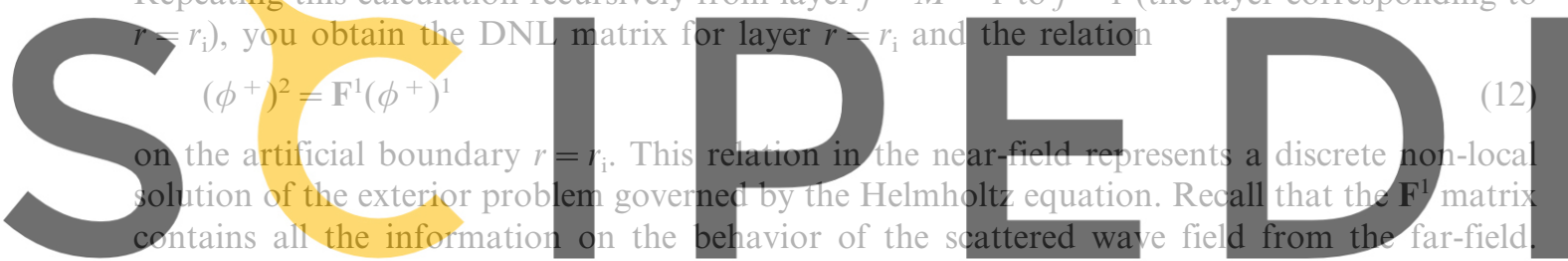

This process is numerically stable and is called 'condensation from the plane DNL matrix to the circular DNL matrix'. This process is numerically stable and is called 'condensation from

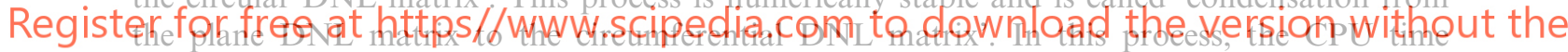

increases with the exterior radius $r_{\mathrm{e}}$, without increasing the RAM memory. Recall that, in general, thees kind of applications are memory bounded. Furthermore, the number of operations can be drastically reduced by means of an eigenvalue decomposition of the matrix $\mathbf{M}^{-1} \mathbf{K}$.

\section{REFLECTION COEFFICIENT IN THE FAR-FIELD}

This section examines the influence of the location of the $r=r_{\mathrm{e}}$ exterior boundary for each circumferential mode in the solution of the exterior problem (1). The Helmholtz equation in circumferential co-ordinates on the annular region is reduced by a Fourier transformation in a Bessel equation of order $n$, where $n$ represents the circumferential mode order. In the 'condensation' procedure, the location of $r=r_{\mathrm{e}}$ is considered sufficiently far. In virtue of this, the curvature effects are depreciable. For $r \geq r_{\mathrm{e}}$, a good approximation to the $\phi^{\mathrm{s}}$ solution of the Bessel equation of order $n$ is the asymptotic expression [20] 


$$
\phi^{\mathrm{s}} \sim \exp \left[i \sqrt{k^{2}-\left(\frac{n}{r_{\mathrm{e}}}\right)^{2}} r\right]
$$

yielding the condition

$$
\frac{\mathrm{d} \phi^{\mathrm{s}}}{\mathrm{d} r}-i \sqrt{k^{2}-\left(\frac{n}{r_{\mathrm{e}}}\right)^{2}} \phi^{\mathrm{s}}=0 .
$$

Employing the exact solution of the Helmholtz equation by means of the representation

$$
\phi^{\mathrm{s}}(r, \theta)=\left(H_{n}^{(1)}(k r)+\operatorname{Ref} H_{n}^{(2)}(k r)\right) \mathrm{e}^{i n \theta},
$$

you obtain the expression for the reflection coefficient $\mid$ Ref $\mid$

$$
|\operatorname{Ref}|=\frac{\left|\left(H_{n}^{(1)}(k r)\right)_{r}^{\prime}-i \sqrt{k^{2}-\left(\frac{n}{r_{\mathrm{e}}}\right)^{2}}\left(H_{n}^{(1)}(k r)\right)\right|}{\left|\left(H_{n}^{(2)}(k r)\right)_{r}^{\prime}-i \sqrt{k^{2}-\left(\frac{n}{r_{\mathrm{e}}}\right)^{2}}\left(H_{n}^{(2)}(k r)\right)\right|},
$$

along $r=r_{\mathrm{e}} \cdot H_{n}^{(1)}$ is the Hankel function of the first kind and order $n . H_{n}^{(2)}$ is the Hankel function of the second kind and order $n$.

Figure 3 shows the curves of reflection coefficients with respect to the progressive modes for the condensation widths given. Several condensation widths have been selected. Note that when the condensation width increases, the reflection coefficient |Ref| diminishes uniformly with respect to $n / k r_{\mathrm{e}}$. In this figure, it can observed that the reflection coefficient in the far-field is always less than 0.05 . For the first modes, the reflection coefficient is less than 0.005. Also its noted that the reflection coefficient diminishes for each circumferential mode. If a 'condensation' width of $8 \lambda$ is selected, the curves drawn represent the circumferential modes for a reflection coefficient Ref whose value is less than 0.005 for $n / k r_{\mathrm{e}} \leq 0.9$
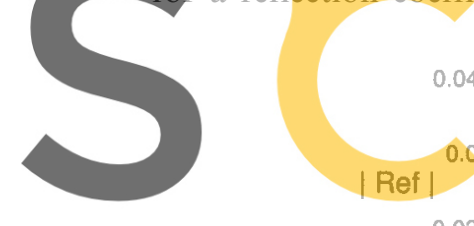

\section{Register for free}

\section{ht}

htt.tgs
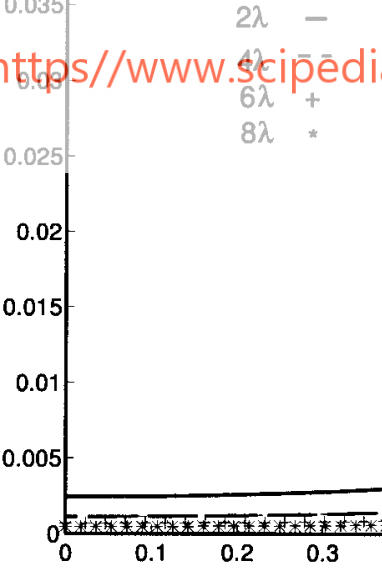

$2 \lambda-$

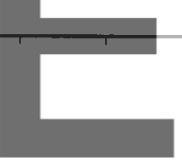

$6 \lambda^{+}$

$8 \lambda *$

ia.co 


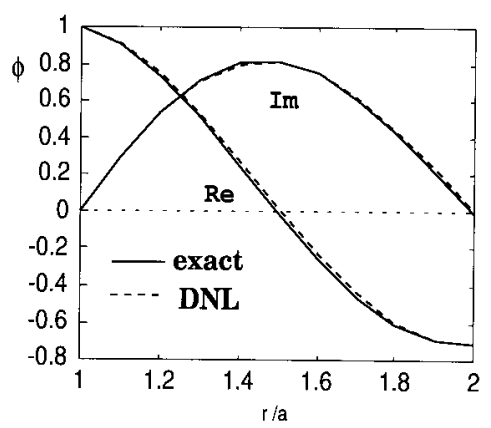

Figure 4. Uniformly pulsating cylinder of radius $a, r_{\mathrm{i}}=2 a, k a=\pi$.

It may be expected that scattering errors along the $r=r_{\mathrm{i}}$ boundary will diminish as the circle $r=r_{\mathrm{e}}$ is placed far away from the computational domain. The appropriate condensation width has been sought for a given predetermined reflection coefficient.

\section{NUMERICAL RESULTS}

All numerical experiments are performed on two-dimensional geometries of problems representing infinite cylinders of radius $a$. DNL boundary conditions are imposed on the artificial boundary at radius $r_{\mathrm{i}}$. The domain is discretized by linear quadrilateral elements, with 60 elements in a circumferential layer. The number of element layers is selected so that the

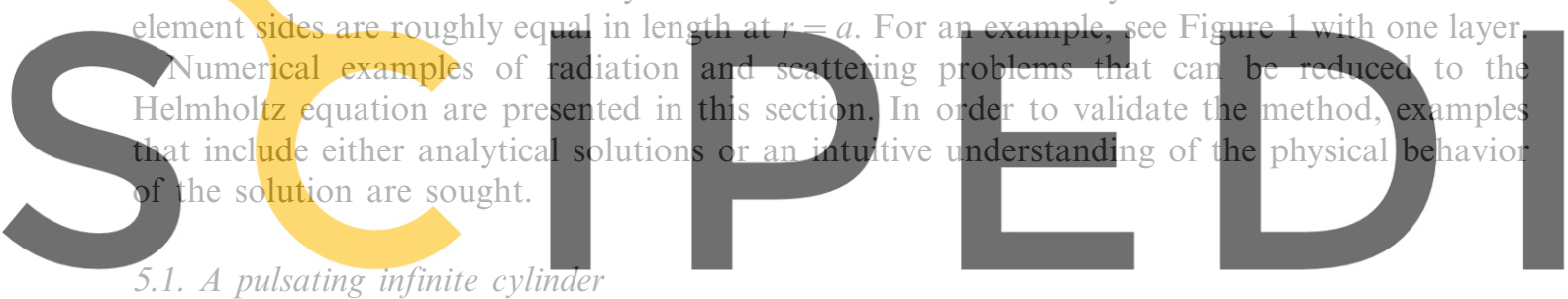

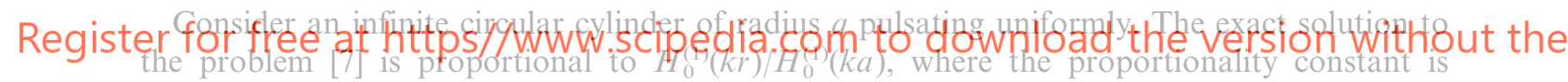

adjusted to satisfy the boundary conditions on the physical boundary. The artificial boundary is located at $r_{\mathrm{i}}=2 a$, and the resulting computational domain is discretized with linear quadrilateral elements.

Problems with Dirichlet boundary conditions on the wet surface are considered, in the region in which the exact solution is a propagating cylindrical wave. The numerical results preserve the cylindrical symmetry of the exact solution, and are presented along a ray. For a resolution of 20 elements per wave (the wavelength is equal to the diameter of the cylinder) the Galerkin solution exhibits a good correspondence with the exact solution in both amplitude magnitude and phase magnitude, as observed in Figure 4.

\subsection{Circumferentially harmonic radiation from a cylinder}

Next, the same problem is considered but with a load distribution $\cos n \theta$, where the exact solution [7] is $H_{0}^{(1)}(k r) \cos n \theta / H_{0}^{(1)}(k a)$. The previous computational configuration is retained, with $r_{\mathrm{i}}=2 a$ and 20 linear quadrilateral elements. The fifth circumferential mode $n=4$ and a 


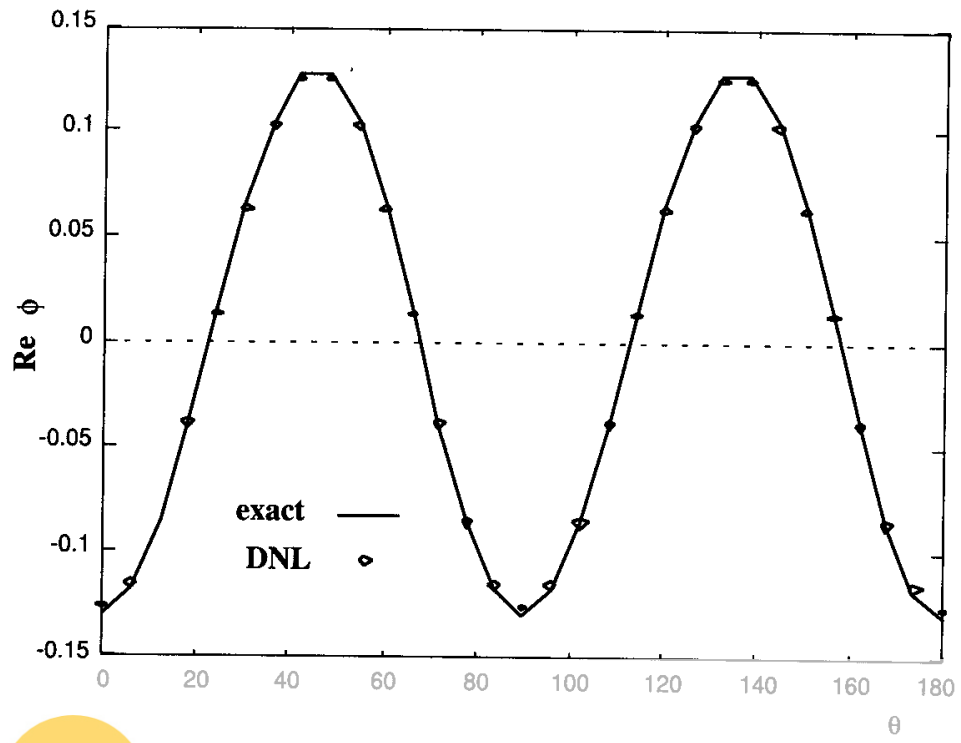

Figure 5. Circumferentially harmonic $(n=4)$ radiation from a cylinder of radius $a, k a=\pi$, along the artificial boundary $r_{\mathrm{i}}=2 a$.

geometrically non-dimensionalized wavenumber $k a=\pi$ have been examined (the wavelength is equal to the diameter of the cylinder). Figure 5 shows the real parts of the exact solution and numerical solution along the artificial boundary $r_{\mathrm{i}}=2 a$. Figure 6 depicts the imaginary parts

of the exact solution and numerical solution aleng the artificial boundary $r_{\mathrm{i}}=2 a$.

Employing a condensation process with eight vavelengths, an excellent correspondence is obtained between the exact and numerical solutions along the artificial boundary $r_{\mathrm{i}}=2 \boldsymbol{\alpha}$, as is
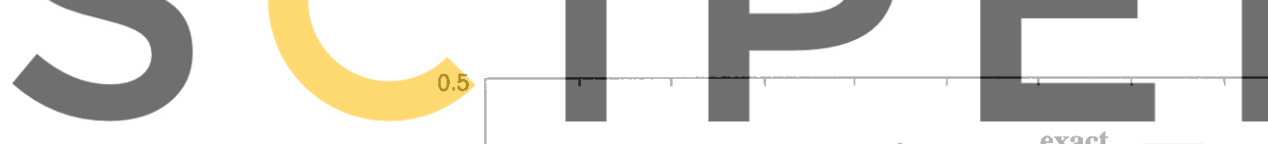

Register for free at httips Xwww.scipedia.com to downifoad the version without the

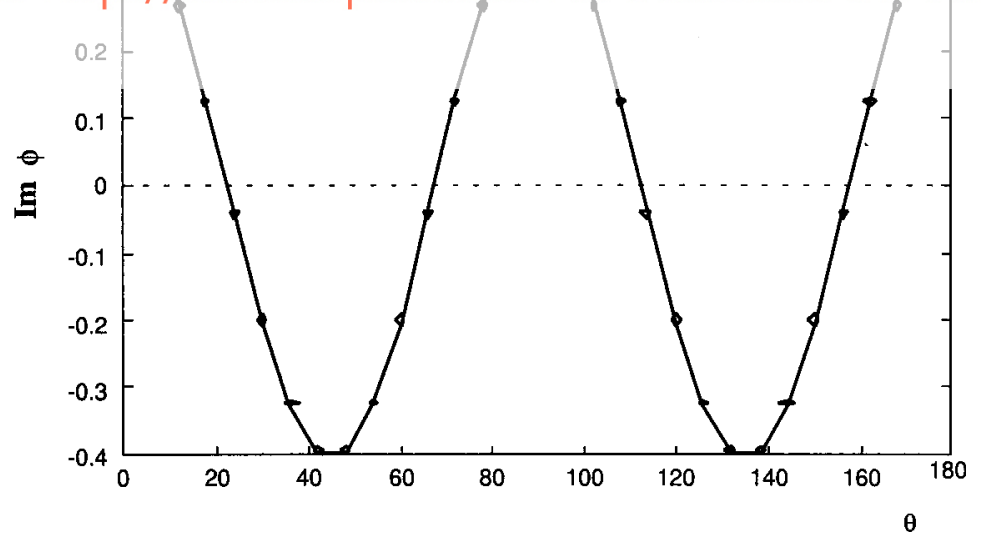

Figure 6. Circumferentially harmonic $(n=4)$ radiation from a cylinder of radius $a, k a=\pi$, along the artificial boundary $r_{\mathrm{i}}=2 a$. 

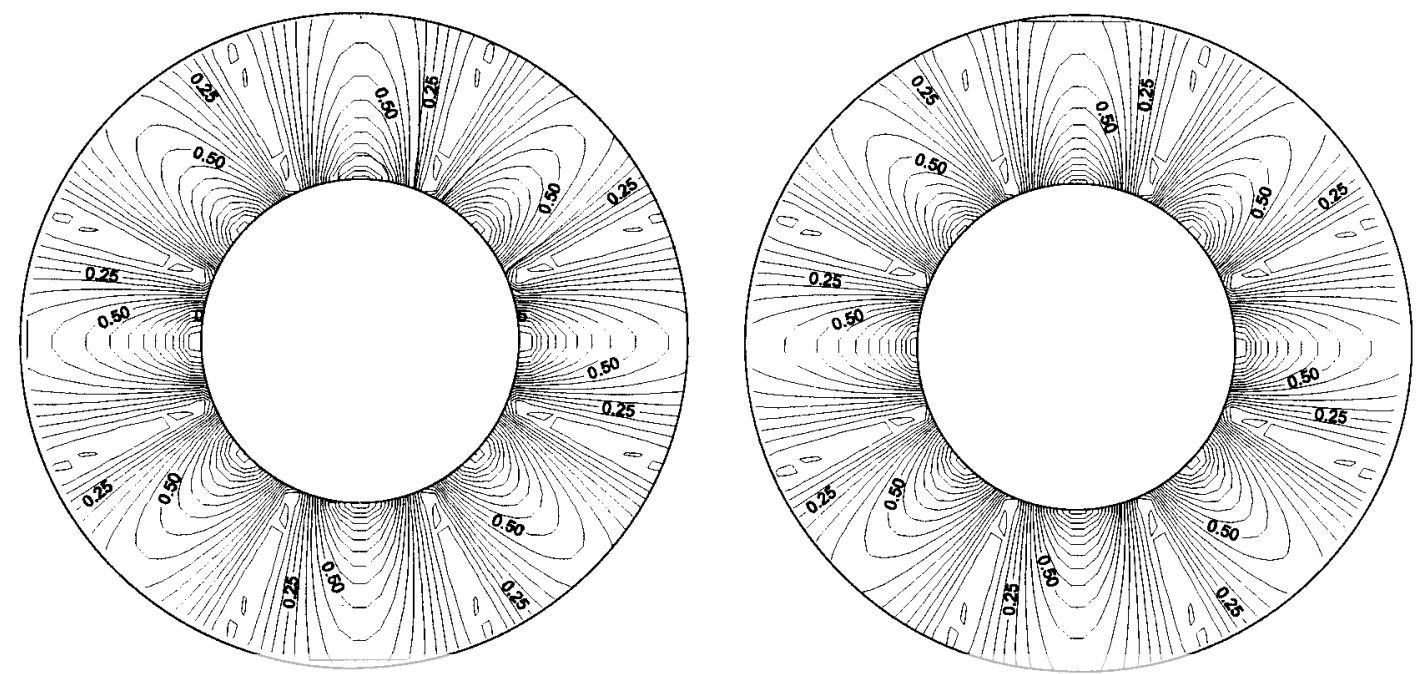

Figure 7. Circumferentially harmonic $(n=4)$ radiation from a cylinder of radius $a, k a=\pi$. Absolute values of exact solution (left) and numerical solution (right).

presented in Figures 5 and 6. These numerical results have been obtained with a reflection coefficient $\mid$ Ref $\mid=0.004729$ in the far-field, including the first five modes [16]

Figure 7 shows the capability of Galerkin methods in combination with the DNL boundary condition to model loads that change rapidly in the circumferential direction.

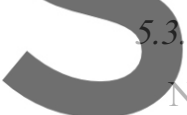

Radiation from an element of a cylinder Now, a harder problem is

considered.

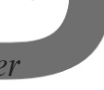

infinite circular cylinder with a constant inhomogeneous value on an arc $(-\alpha<\theta<\alpha)$ and vanishing elsewhere. The normalized analytical solution to this problem for a cylinder of Registerder free at https//www.scipedia.com to download the version without the

$$
\phi(r, \theta)=\frac{2}{\pi} \sum_{n=0}^{\infty}, \frac{\sin (n \alpha)}{n} \frac{H_{n}^{(1)}(k r)}{H_{n}^{(i)}(k a)} \cos (n \theta) .
$$

For low wavenumbers this solution is relatively uniform in the circumferential direction. The directionality of the solution grows as the wavenumber is increased, and the solution becomes attenuated at the side of the cylinder opposite to the radiating element.

The properties and discretization are unchanged from the previous problem. A value of $\alpha=5 \pi / 32$ has been selected and a condensation width of eight wavelengths has been employed.

Figure 8 (left) depicts the imaginary part of the solutions evaluated along the artificial boundary $r_{\mathrm{i}}=2 a$. Figure 9 shows the absolute values of the analytical solution, nodally interpolated by the mesh employed. The low amplitude oscillations in the vicinity of the wet surface are merely a product of the series representation of the discontinuity in the boundary condition, and they are not relevant to the validation of the numerical results. The numerical solution captures the essential physics of the problem, but their individual performance is difficult to evaluate from the contour plots. 


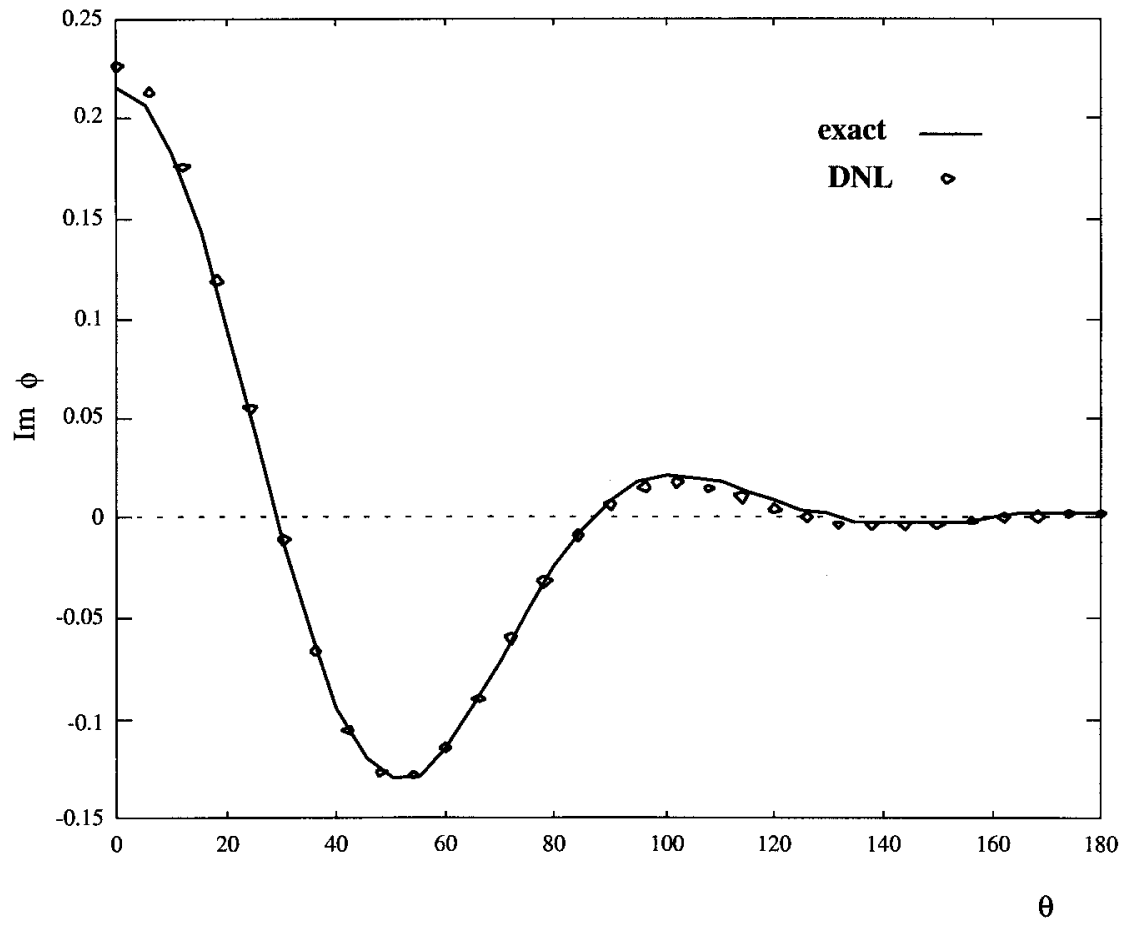

Figure 8. Radiation from an element of a cylinder of radius $a, k a=\pi$, along the artificial boundary $r_{\mathrm{i}}=2 a$. Imaginary part of numerical solutions for $N_{\text {lay }}=60$.

\subsection{Scattering of a plane wave from a cylinder}

The difference between an undisturbed wave and the field generated when the wave encounters an obstacle is called a scattered wave. In the typical range of this phenomena, the
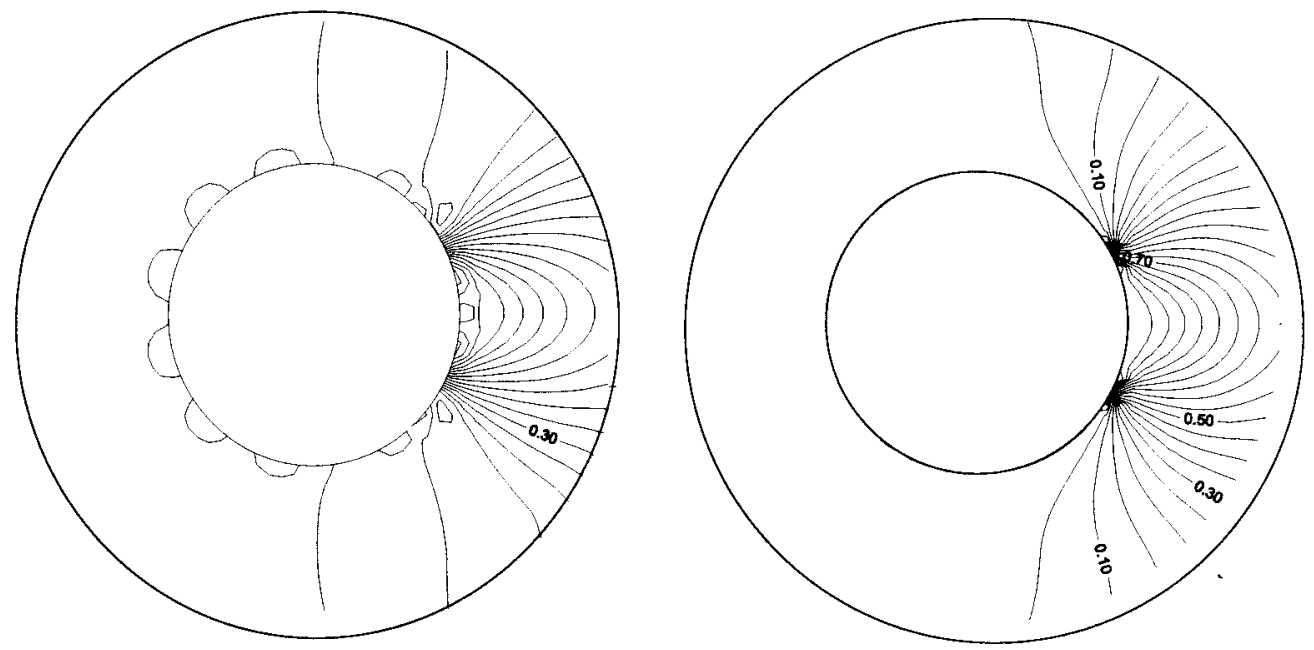

Figure 9. Radiation from an element of a cylinder of radius $a, r_{\mathrm{i}}=2 a, k a=\pi$. Nodal interpolation of the series solution (left); Galerkin solution with DNL condition for $N_{\text {lay }}=60$ (right). 


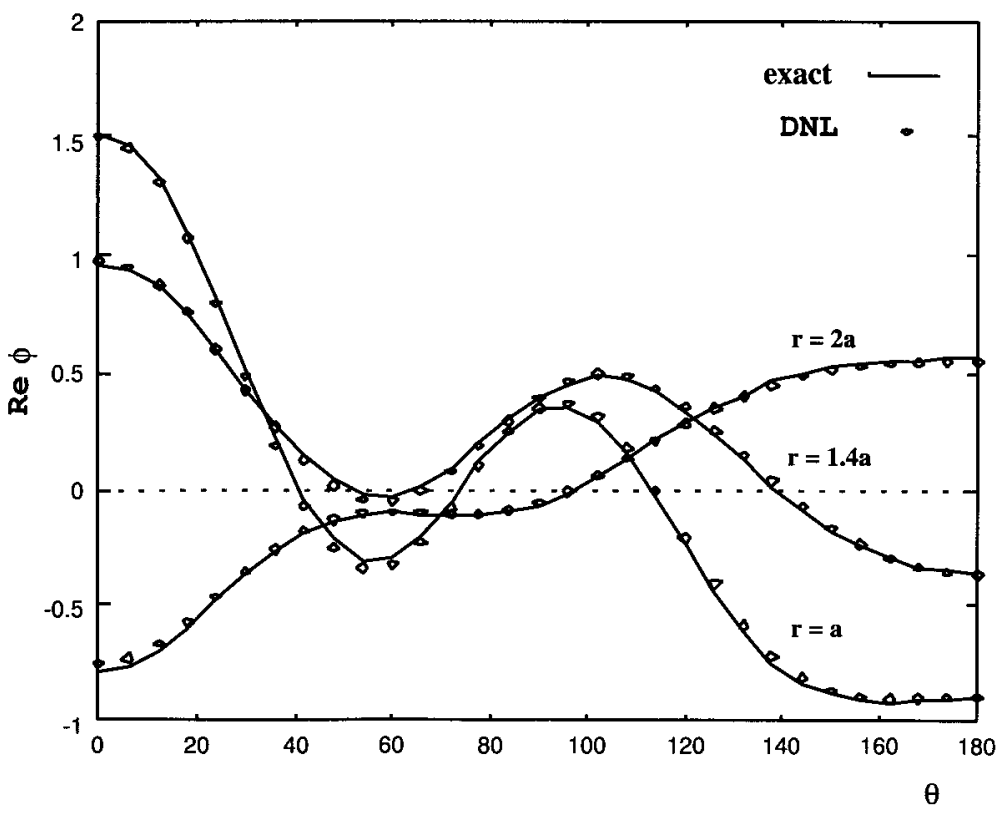

Figure 10. Scattering of a plane wave (at $\theta=0$ ) from a cylinder of radius $a, k a=\pi$. Real parts of the Galerkin solution at $r=a, 1.4 a, 2 a$.

scattered wave usually does not destructively interfere with the incident wave, allowing their complete separation. As an example, the scattering by a cylinder, of radius $a$, of an incident plane wave traveling along the positive $x$-axis $(\theta=0)$ in a normal direction to the cylinder's axis has been computed. The analytical solution of the diffraction around a circular pile (see Figure 12 (left)) has been given by McCamy and Fuchs [1] with the aid of a Bessel series expansion. The normalized analytical solution to this problem is

$$
\phi(r, \theta)=\sum_{n=0}^{\infty} \epsilon_{n} i^{n} A_{n}(k r) \cos (n \theta) \quad \epsilon_{0}=-1, \quad \epsilon_{n}=-2 \quad \text { for } n=1,2,3, \ldots
$$

where

$$
A_{n}(k r)=\frac{J_{n}^{\prime}(k a) H_{n}^{(1)}(k r)}{H_{n}^{(1) \prime}(k a)} .
$$

Again, the directionality of the response increases with wavenumber, and the distribution becomes more complicated. The properties and discretization are retained from the previous problems considered. A hard boundary on the wet surface to represent a rigid solid was selected, and again a condensation width of eight wavelengths employed. Figures 10 and 11 show the real and imaginary parts of the Galerkin solution respectively, for different values of radius $r=a, 1.4 a$ and $2 a$. In both figures, the numerical solutions represent the expected physical behavior of the solution. Figure 12 shows absolute values of the analytical/numerical solution respectively. Again, the excellent agreement between them is clear. 


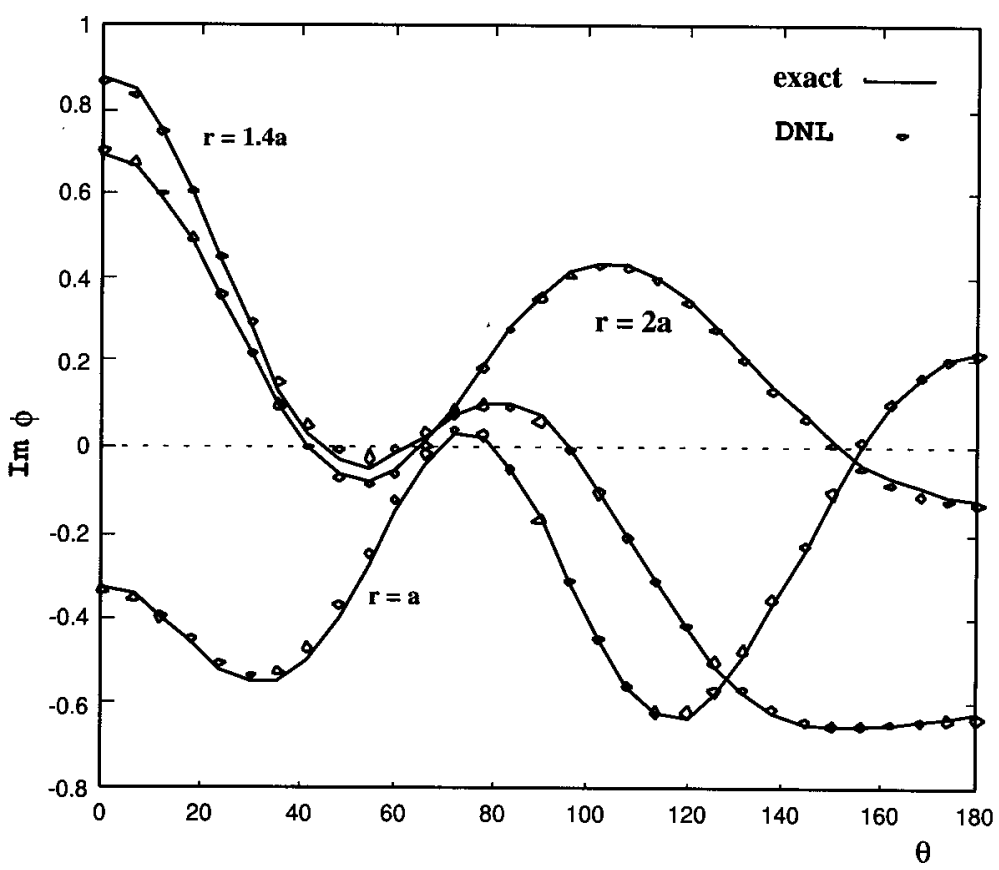

Figure 11. Scattering of a plane wave (at $\theta=0$ )from a cylinder of radius $a, k a=\pi$. Imaginary parts of the Galerkin solution at $r=a, 1.4 a, 2 a$.

\section{COMPARING DNL WITH LOCAL BOUNDARY CONDITIONS}

Various boundary conditions on the artificial boundary will be numerically compared with the DNL boundary condition by considering non-uniform radiation from a rigid infinite circular cylinder with circumferentially harmonic loading. Preserving the physical characteristics mentioned in Section 5.2, a mesh of 24 elements has been used for an annular region limited by
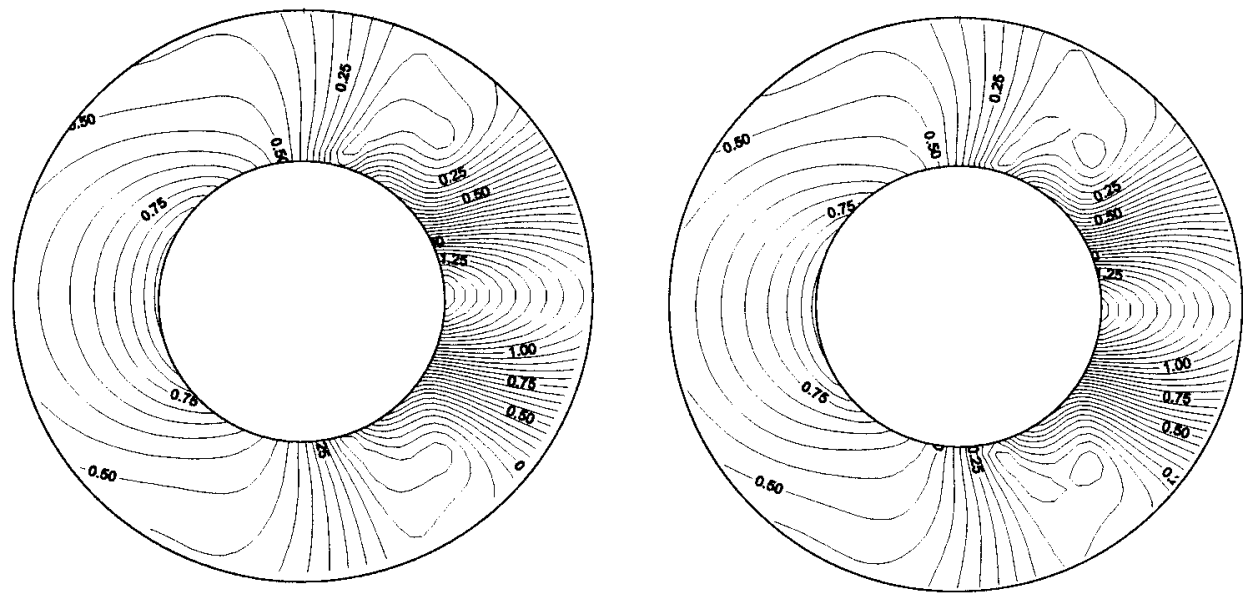

Figure 12. Scattering of a plane wave (at $\theta=0$ )from a cylinder of radius $a, k a=\pi$. Absolute values of the nodal interpolation of the series solution (left) and the Galerkin solution (right). 


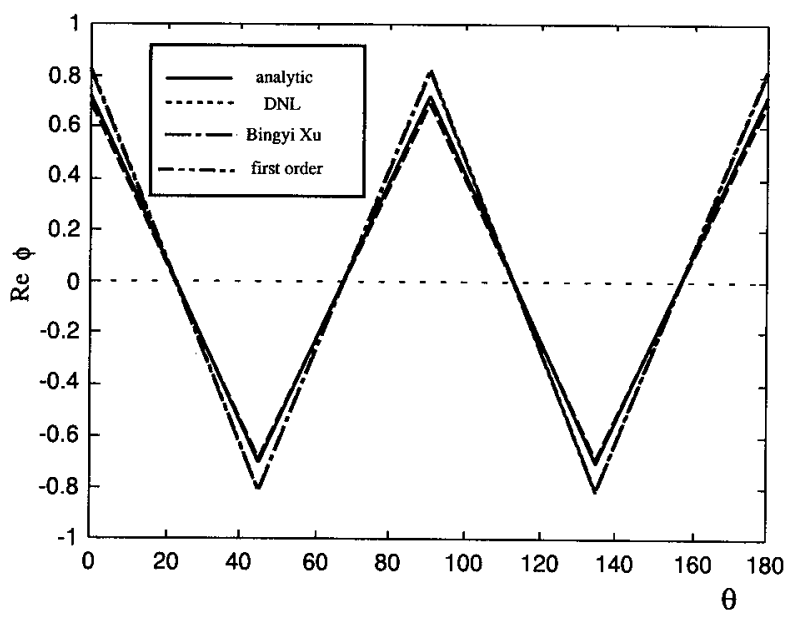

Figure 13. Circumferentially harmonic radiation from a cylinder of radius $a, r_{\mathrm{i}}=1.2 a, k a=\pi$ Comparing DNL with several local boundary conditions for $N_{\text {lay }}=8$. Real parts of numerical solutions.

$1.0 a \leq r<1.2 a$. Close to the limit of resolution, the solution for a mesh with $\approx 2.5$ elements per wave has been computed, in which the Galerkin solution has a significant degradation. Figures 13 and 14 show the comparison between the real parts and the imaginary parts of the different numerical solutions obtained with the Galerkin method in combination with such conditions at the artificial boundary $r=1.2 a$. As expected, the DNL solution is superior to other boundary conditions considered.

With the objective of an exhaustive evaluation of this criterion, the relative errors between the analytical solution and numerical solutions are calculated in two layers of element (an interior layer and on the boundary layer). The first case corresponds to the radio $r=1.1 a$, and the second case corresponds to the case $r=1.2 a$. Table I shows the corresponding maximum relative error values. In the second and third columns, the maximum relative error diminishes

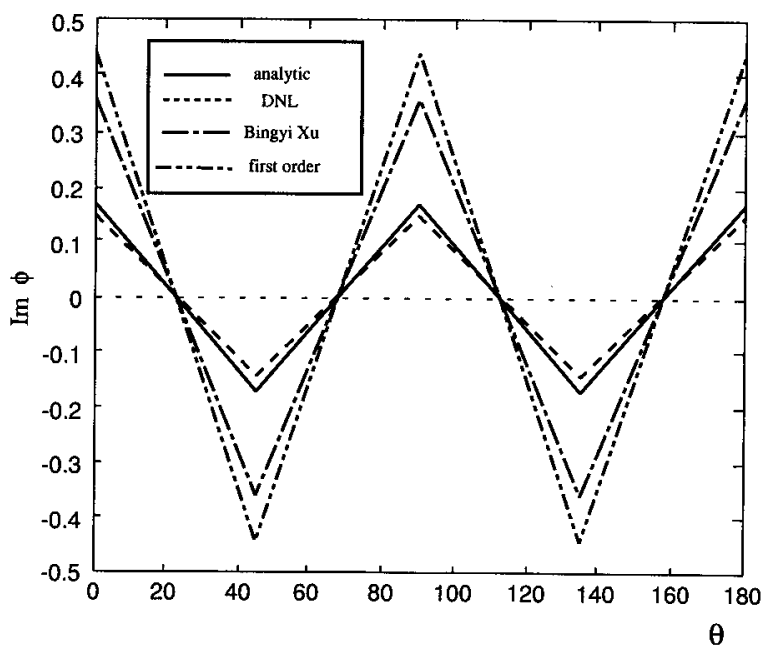

Figure 14. Circumferentially harmonic radiation from a cylinder of radius $a, r_{\mathrm{i}}=1.2 a, k a=\pi$. Comparing DNL with several local boundary conditions for $N_{\text {lay }}=8$. Imaginary parts of numerical solutions. 
Table I. Maximum relative error values (\%)

\begin{tabular}{lcc}
\hline Relative error (\%) & $r=1.1 a$ & $r=1.2 a$ \\
\hline First-order b.c. & $16.7 \%$ & $38.0 \%$ \\
Second-order b.c. & $13.0 \%$ & $30.0 \%$ \\
DNL b.c. & $3.5 \%$ & $4.5 \%$ \\
\hline
\end{tabular}

accordingly as the complexity (non-locality) of the b.c. increases. The minimum values correspond to the DNL method. The improvement of the DNL solution in comparison with the others is striking in this case.

\section{CONCLUSIONS}

In this paper, we have developed a finite element method for $2 \mathrm{D}$ exterior boundary value problems governed by the Helmholtz equation. Galerkin methods in combination with DNL boundary conditions represents a general purpose methodology for these kind of problems. In contrast to the DtN formulation, the DNL formulation may be implemented as a 'black box' numerical routine, which computes the boundary absorbing matrix $\mathbf{F}$ in terms of the standard FEM coefficients matrices: $\mathbf{A}, \mathbf{B}$ and $\mathbf{C}$, independent of the particular operator at hand. Numerical studies show that there are no restrictions on the location of the artificial boundary $r=r_{\mathrm{i}}$. The new procedure hence, enables the modeler to relax the requirement on the geometry to some extent. However, the condensation process is still limited to a constant wavenumber outside of $\mathscr{B}$.

We have analyzed in detail numerical solutions to model problems describing physical phenomena that include propagation, in which the accuracy of the numerical solutions show that the DNL method represents the far-field adequately.

The DNL method does not add any virtual complexity in the implementation. The computation of the eigenvalue decomposition is based on the LAPACK routine package for the determination of eigenvalues. The computational process in circumferential co-ordinates does not represent any excessive amount of RAM memory, because the DNL matrix in each layer is not stored.

\section{ACKNOWLEDGMENTS}

This work has received financial support from Consejo Nacional de Investigaciones Científicas y Técnicas (CONICET, Argentina) through grant BID 802/OC-AR PID Nr. 26, and from Universidad Nacional del Litoral (Argentina). The authors made extensive use of software distributed by the Free Software Foundation/GNU-Project: Linox ELF-OS, Octave from J.W. Eaton, Tgif from W.C. Cheng, Fortran f2c compilers, and others.

\section{APPENDIX A. NOMENCLATURE}

a radius of cylinder

$\mathbf{A}^{j} \quad$ matrix corresponding to $j-1$ layer 


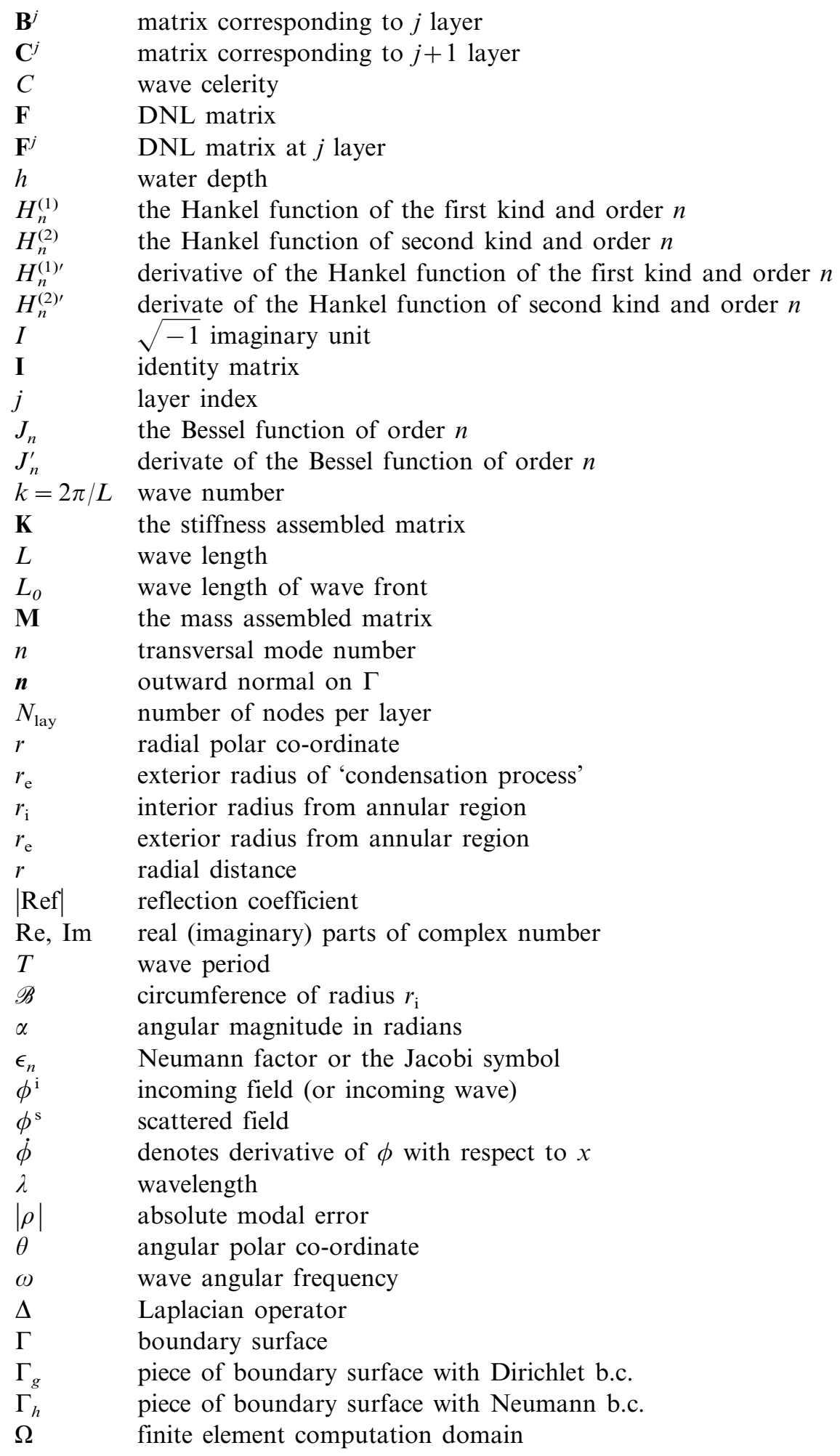




\section{REFERENCES}

1. J.C.W. Berkhoff, 'Mathematical models for simple harmonic linear water waves. Wave diffraction and refraction', Publication No. 163, Delft Hydraulic Laboratory, 1976.

2. J.C.W. Berkhoff, N. Booy and A.C. Radder, 'Verification of numerical wave propagation models for simple harmonic linear water waves', Coast. Eng., 6, 255-279 (1982).

3. G. Chertock, 'Integral equation methods in sound radiation and scattering from arbitrary structures', NSRDC Tech. Rep. 3538, Washington, DC, 1971.

4. H.S. Chen, 'Infinite elements for water wave radiation and scattering', Int. J. Numer. Methods Fluids, 11, 555-569 (1990).

5. A. Bayliss and E. Turkel, 'Radiation boundary conditions for wave-like equations', Commun. Pure Appl. Math., 33, 707-725 (1980).

6. R.A. Dalrymple and P.A. Martin, 'Perfect boundary conditions for parabolic water wave models', Proc. R. Soc. Lond., A437, $41-54$ (1992).

7. D. Givoli, Numerical Methods for Problems in Infinite Domains, Elsevier, Amsterdam, 1992.

8. I. Harari and T.J.R. Hughes, 'Studies of domain-based formulations for computing exterior problems of acoustics', Int. J. Numer. Methods Eng., 37, 2935-2950 (1994).

9. J.M. Grassa, Parabolic Models for Propagation of Water Waves, CEPYC, CEDEX, Madrid, Spain (in Spanish), 1991.

10. J.T. Kirby, 'A note on parabolic radiation boundary conditions for elliptic wave calculations', Coast. Eng., 13, 211-218 (1989).

11. B. Xu, V.G. Panchang and Z. Demirbilek, 'Exterior reflections in elliptic harbor wave models', J. Waterw. Port Coast. Ocean Eng., 122, 118-126 (1996).

12. R.P. Bonet, N. Nigro, M.A. Storti and S.R. Idelsohn, 'Non-local absorbing discrete boundary condition (DNL) in finite difference for water waves elliptic models', (in Spanish), accepted for publication in RIMNE (Revista Internacional de Mètodos Numéricos para el Càleulo y diseño en Ingenieria) (1997).

13. R.P. Bonet, N. Nigro, M.A. Storti and S.R. Idelsohn, 'Non-local absorbing discrete boundary condition (DNL) in finite elements for water waves elliptic models', (in Spanish), accepted for publication in RIMNE (Revista Internacional de Mètodos Numéricos para el Càleulo y diseño en Ingenieria) (1997).

14. D. Givoli and J.B. Keller, 'A finite element method for large domains', Comput. Methods Appl. Eng., 76, 41-66 (1989).

15. D. Givoli and J.B. Keller, 'Non-reflecting boundary conditions for elastic waves', Wave Motion, 12, 261-279 (1990).

16. I. Harari and T.J.R. Hughes, 'Galerkin/least-square finite element methods for the reduced wave equation with non-reflecting boundary conditions in unbounded domains', Comput. Methods Appl. Mech. Eng., 98, 411-454 (1992).

17. Y. Chen and P.L.-F. Liu, 'A pseudo-spectral approach for scattering of water waves', Proc. R. Soc. Lond., A445, 619-636 (1994).

18. R.W. Thatcher, 'The use of infinite grid refinements at singularities in the solution of Laplace's equation', Numer. Math., 25, 163-178 (1976).

19. R.W. Thatcher, 'On the finite element method for unbounded regions', SIAM J. Numer. Anal., 15, 456-477 (1978).

20. A.N. Tijonov and A.A. Samarsky, Equations of the Physica-Mathematica (translation to Spanish), Edit MIR, Moscow, 1980. 